\title{
Tools for Strategic Decision Support in Energy Sector Based on Situation Management and Semantic Modeling
}

\author{
Liudmila V. Massel1 ${ }^{1}$, Vladimir R. Kuzmin ${ }^{2}$ \\ Melentiev Energy Systems Institute of Siberian Branch of the Russian Academy of Sciences, \\ Lermontov str., 130 \\ Irkutsk, Russia \\ ${ }^{1}$ E-mail: massel@isem.irk.ru \\ ${ }^{2}$ E-mail: kuzmin_vr@isem.irk.ru
}

\begin{abstract}
Team, represented by authors, develops a direction, related with support of strategic decision-making in energy sector based on concepts of situation management and semantic modeling. The architecture of instrumental system, called Situational polygon, which is designed for decision-making support is shown. One of the main components of this system is Contingency Management Language or CML (with main focus on management in critical situations conditions in energy). This article deals with approaches to implementation of CML, based on usage of situation calculus and special software complex Semp-TAO.
\end{abstract}

Keywords: Situational polygon, semantic modeling, Semp-TAO, intelligent decision-making support system, contingency management language, situation calculus.

\section{Introduction}

Problem of intelligent support of decision-making is truly relevant, especially due to spread of the concept on the intelligent energy system in Russia [1]. The authors of paper [2] say that due to the specific economic situation in Russia the Smart Grid concept has become more popular there. Federal Grid Company of Unified Energy System, like most power companies in Russia has chosen the Smart Grid concept of "Intelligent System" implementation. Moreover, this company tries to support and finance national equipment and material manufacturers; they have a special program for them. Also, some pilot projects in Siberia, in the Far East, in North West are realized with the support of this company. However, implementation of this concept requires (1) improvement of technological infrastructure, (2) development and (3) application of modern information technologies to manage it. The first one is directly connected with strategic decision-making in developing energy and demands intellectualization of tools to support decisionmaking.

The semiotic approach proposed by the authors integrates the methods of situation management, semantic modeling and cognitive graphics.

\section{State of Art}

The situation calculus has been widely applied in the multi-agent modeling. Thus, in [3] the authors discuss the possibility to employ a new approach to deriving new knowledge in multi-agent systems to eventually let an agent act with available data only, instead of inquiring knowledge from all agents. In paper [4] they describe the approach to verify temporal properties of agents possessing incomplete knowledge on the world and using situation calculus bounded action theories. Besides, in [5] the researchers proposed to employ situation calculus and event calculus in the general theory of steady models and answer set programming. In [6] the research team reports creating the prototype language of programming to manage the YAGI robot. YAGI has been designed with Golog language involved and based on situation calculus.

In our case semantic modeling is targeted to integrate various sources of data. Article [7] supports usage of semantic technologies for designing models of urban electric networks. A semantic energy information framework brings together the data sources at different scales and from different domains, as well as some energy simulation and assessment tools that interact with the semantically modeled data. An ontology based on a vocabulary of concepts shared by experts has been 
created through the following process: capturing the experts knowledge about a specific problem regarding the energy efficiency of an urban area and the data needed to model it; creating an informal vocabulary through the terms referred to technical standards, and creating a formal vocabulary according to the Ontology Web Language specifications. The authors of paper [8] employed semantic modeling of engineering data for creating the ontologies capable to extract data for intellectual engineering applications. Whereas in [9], they suggest to apply semantic approach for searching and visualizing academic information.

Since 2013, the authors of this article have been supporting semantic technologies for cognitive, eventtrigger and probabilistic (based on the Bayesian networks of confidence) modeling [10].

Semiotics is the science studying the properties of signs, signed and symbol systems used through process of communication. It has been currently intensely developed worldwide [11].

The semiotic approach to constructing the intellectual systems was proposed by Russian scientist D.A. Pospelov, who believed, that the elements of sign or semiotic systems investigated in semiotics can vary, and the variability and conventionality of signs would let the effective intellectual systems to be created.

The semiotic approach is applicable for developing the system to plan the behaviour and routes of robots. These robots are considered as the agents sharing the same goal acting in the 2D space, which might change in time [12]. In article [13] the researchers show the possibility to stick to the semiotic approach in critical reasoning.

It is noteworthy, that the authors have not found the information on the development of utilization of situation calculus in power engineering and with reference to situation management systems, as well as data on the efforts to utilize the situation calculus in the field of situation management.

Now, we will main concepts of situation management, semiotic approach and situation calculus into closer consideration.

\section{Situation Management}

Term "situation management" was introduced in the 1960's by Pospelov D.A, the founder of this trend. Initially, term "model management" was used, this concept was actively developed by Pushkin V.N. and Pospelov D.A. Then, the concept "situation model" was introduced. With the advent of the Pospelov D.A.'s article [14] the term "situation management" replaces all the rest.
In modern articles, situation management is interpreted as follows. The aggregation of the current state of the object (state vector $\mathbf{X}$ ) and its external environment (disturbance vector $\mathbf{F}$ ) stands for current situation $\mathbf{C}$. Then, $\mathbf{C}=\langle\mathbf{X}, \mathbf{F}\rangle$. Concept of full situation is being introduced: $\mathbf{S}=\langle\mathbf{C}, \mathbf{G}\rangle$, where $\mathbf{C}-$ current situation, $\mathbf{G}$ - management goal. Then, management goal $\mathbf{G}$ may be represented as goal situation $\mathbf{G g}$, to which current situation must be adjusted. Thus, $\mathbf{S}=\langle\mathbf{C}, \mathbf{G g}\rangle$. Assuming that current situation $\boldsymbol{C}$ is a part of some class $\boldsymbol{Q}^{\prime}$, and goal situation $\boldsymbol{G}_{\boldsymbol{g}}$ is a part of $Q^{\prime \prime}$, it is critical to find such management (control vector $\boldsymbol{U}$ ), which belongs to a number of admissible actions $\boldsymbol{\Omega}_{\mathbf{u}}$ and provides required transformation of one class of situations to the other:

$$
C \in Q^{\prime} \stackrel{U \in \Omega_{u}}{\longrightarrow} G_{g} \in Q^{\prime \prime}
$$

So, situation management appears as a relation:

$$
\left(Q^{\prime}, Q^{\prime \prime}\right) \rightarrow U \in \Omega_{u},
$$

which matches pair "current situation - goal situation" with the desired result - management $\mathrm{U}$.

In terms of situation management the energy security is thought to be the management of extreme situations in the energy sector or contingency management. Under term "extreme situations" both critical and emergency situations are pictured in accordance with scale "normal - precrisis - crisis", the critical situation being particularly stressed upon [10]. The concept is primarily employed for situation management of the Russian energy systems and fuel and the sector in general, with special focus on the requirements to energy security [15].

\section{Semiotic approach}

In areas close to the informatics semiotics is used in such areas as data processing, algorithms that provide processing of texts in natural language, artificial languages, compilation of cartographic images, special schemes and plans. It is noted by leading semiotics scientists that semiotics has insufficient dissemination in technical areas, or more precisely, lack of works in this areas. The foundations of the semiotic approach to the construction of intelligent systems for the management of technical systems in Russia were laid in the works of Pospelov D.A and Osipov G.S. Pospelov D.A. introduced the definition of semiotic model, which was later detailed by Osipov G.S. [16]. According to Osipov, one can define semiotic model such as:

$$
\begin{aligned}
& W=<T, R, A, P, \tau, \rho, \alpha, \pi>, \text { where } \\
& \mathrm{T}-\text { set of main symbols; } \\
& \mathrm{R}-\text { set of syntactic rules; } \\
& \mathrm{A}-\text { set of facts about subject area; }
\end{aligned}
$$


$\mathrm{P}$ - set of rules for finding solutions (pragmatic rules);

$\tau$ - rules for altering $\mathrm{T}$;

$\rho$ - rules for altering $\mathrm{R}$;

$\alpha$-rules for altering $\mathrm{A}$;

$\pi$ - rules for altering $\mathrm{P}$.

In other words, unlike formal models, where elements that forms set $\mathrm{T}$ has hard syntax, hard semantics and hard pragmatics, in semiotic models all these properties of elements of set $\mathrm{T}$ are available for changes; this is the characteristic feature of signs - the elements of the symbolic, or semiotic, systems studied in semiotics. Such systems are closely related with human activity, it is the variability and conventionality of signs that make this activity effective.

It is worth to note that, semiotic approach in Russia is developing, in particular, in works of Osipov G.S., Vagin V.N., Eremeev A.P. (real time intelligent systems of semiotic type), Kulinich A.A. (semiotic model of cognitive experience) and many others. But in the same time, those works are mostly theoretical.

One of the approaches offered by the authors is to integrate of methods of situation management, which are based on usage of semiotic approach, semantic modeling and situation calculus for development of intelligent systems of semiotic type for decisionmaking support in energetics [10].

Now, the basic concepts of situation calculus will be considered briefly.

\section{Situation Calculus}

In 1963, to describe the model of the world, McCarthy put forward an idea to employ the apparatus of mathematical logics. So he created situation calculus as the formal apparatus for building the world model and reasoning about impacts on this world [17]. The basics of the method are:

- Situation - logical formula that describes complete state of the world at a particular moment in time.

- Fluent - predicates or functions, which values change from one situation to another

- Transition from one situation to another is carried out with special fluent "result".

Purpose of situation calculus is to formalize situations, actions and their causal relationships for some systems. The most common usage area of situation calculus is agent-based modeling [18].

It is proposed to solve two types of problems with situation calculus:

Designing problem - result determination of execution of concrete sequence of actions
Planning problem - finding a sequence of actions letting us achieve certain result [18].

The language of contingency management intuitively assumes that some initial situation exists, and the environment changes from situation to situation while the actions proceed. It is also believed that there are no situations, besides those that can be reached through executing actions. This language is specified by the concepts: categories, predicates and functions [19]

Three disjoint categories are introduced: (a) category of actions A, (b) category of situations S and (c) category of environment objects D. Action category elements are simple or complex constants that denote actions. Complex constants of this category, i.e. constants that are categories, can be contained only in category D elements of. The elements of category of situations are simple constants that denote situations. Elements of the environment category of objects are any simple or complex constants, except for those referred to the actions and situations. Complex constants of this situation can contain only elements from category D.

Standard transition function $A \times S \rightarrow S$. Value of function transition $(a, s)$ is situation $s$ ' into which situation $s$ transforms as a result of action $a . \mathrm{S}_{0}$ is the initial situation. Standard predicate before $\left(s_{1}, s_{2}\right)$ that will be written in normal infix representation $s_{1}<s_{2}$ This predicate is true, if situation $s_{1}$ comes before situation $s_{2} ; s_{1} \leq s_{2}$ means that $s_{1}<s_{2} \vee s_{1}=s_{2}$. Standard predicate allowable $(a, s)$. This predicate is true, if action $a$ is allowed (can be executed) in situation $s$.

Functorial predicate $\varphi\left(d_{1}, d_{2}, \ldots, d_{n}, s\right), \quad$ where $d_{1}, d_{2}, \ldots, d_{n}$ - variables or constants of category $D, \varphi-$ predicate symbol. Functorial predicate contains single argument s, which value belongs to category of situations S. Meaning of the predicate is following: if property or relation $\varphi$ between $d_{1}, d_{2}, \ldots, d_{n}$ take place in situation $\mathrm{s}$, then predicate $\varphi\left(d_{1}, d_{2}, \ldots, d_{n}, s\right)$ is true in this situation and vice versa.

Functorial function $f\left(d_{1}, d_{2}, \ldots, d_{n}, s\right), \quad$ where $d_{1}, d_{2}, \ldots, d_{n}-$ variables or constants of category $D$. Functorial function also contains single argument $s$, which value belongs to category of situations $\mathrm{S}$. Function value belongs to category D.

The key concepts for situation calculus are the objects, object's variables, categories, standard predicates and function, as well as micro situations, states, actions, measures, units and time. Using these concepts, it is feasible to model situations, systematize and classify objects and their features, perform analysis 
of situations and dynamics of their changes and formulate inference rules for the choice of management decisions.

\section{Intelligent Decision-Making Support System "Situational Polygon"}

At the moment, in the laboratory, represented by the authors, an intelligent decision support system "Situation polygon" is being developed [20]. Its architecture is shown on fig. 1. Structure of this system was developed with aim to its implementation as clientserver web application. Work with this application is carried out through the graphical user interface: here user of the system can determine the purpose of working with the system (e.g., search of ontologies in database, work with semantic modeling tools OntoMap, CogMap, etc.). Server side of the application consists of:

- Core - controls user actions, calls semantic modeling tools, works with records in database and Repository.

- Repository - stores models created by knowledge engineers.
- Semantic modeling tools - their work will be implemented with the help of multi-agent technology. Those tools are already implemented by the team represented by authors

- Database - stores system settings and models created by users.

- CML - connects other system components, describes data in the Repository and is used for formation of a sequence of actions.

There will be three users' roles:

- Regular user - has access to semantic modeling tools, models and data in Repository, and also can store his own models in database

- Knowledge engineer - same features as regular user, but additionally he can create and edit models in Repository and has access to all models in database, for purpose of adding some of them to the Repository.

- Administrator - has access to all models in system and also performs administrative functions. Next, we will briefly describe structure of Contingency Management Language.

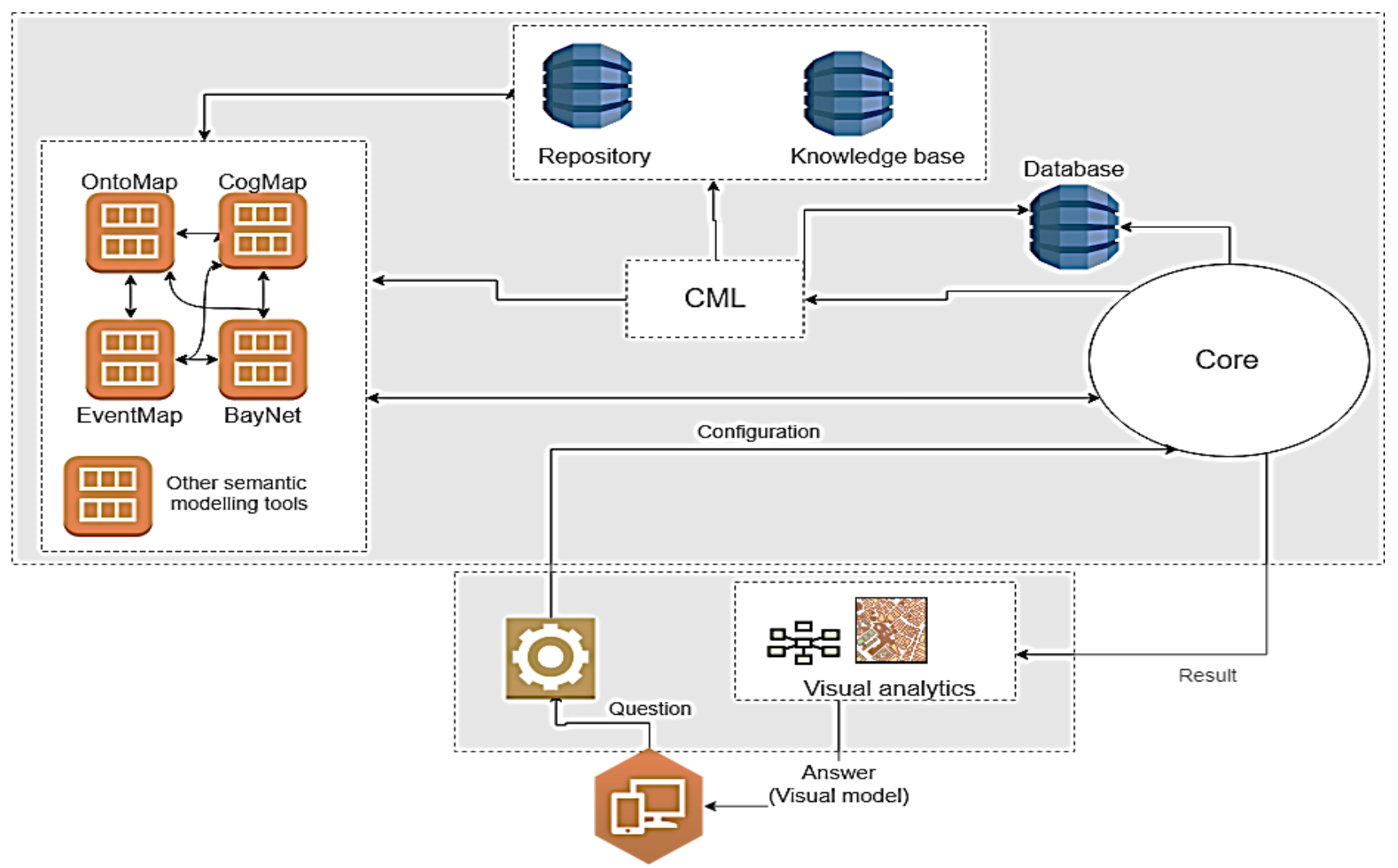

Fig. 1. Architecture of "Situational polygon". 


\section{Contingency management language CML}

CML includes two parts (D, M): tools for knowledge description D (descriptions of situations, scenarios and actions) and tools for knowledge manipulation (support of representations). [21]. Fig. 2 shows metaontology of CML.

Simple construction (XYZ) is intended for knowledge description, where $\mathrm{X}$ and $\mathrm{Z}$ are the terms or names, and $\mathrm{Y}$ is the relation or action.

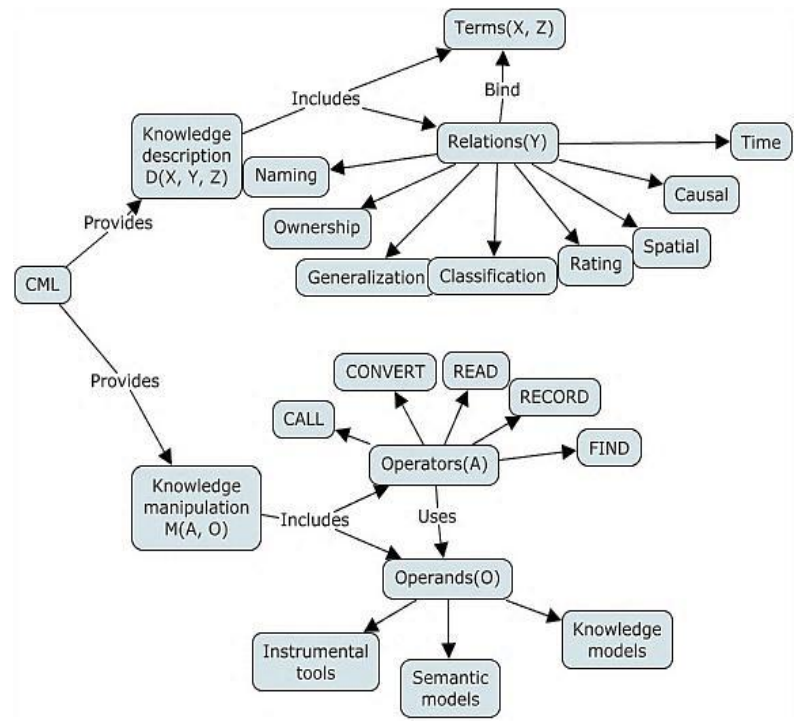

Fig. 2. CML Metaontology.

Now, we will consider two approaches and how it is possible to utilize them for development of CML.

\section{Situation calculus as development of semiotic approach}

Situation calculus is the tool for situation formalization in dynamic state to fix up a combination of facts and properties of objects and environment, in which these objects operate. It is a formal system, based on the firstorder predicate logic, so, it can be considered as the logical language to describe dynamics of environment change. With this formal language it is feasible to express all knowledge about the environment, the subject area, which is required for solving the proposed task. Semantics define the meaning of these sentences. Symbols are compared with the objects of real world, and the sentences correspond to the relations between objects.

The language of situation calculus intuitively assumes that some initial situation exists, and the environment changes from situation to situation while the actions proceed. It is also believed that there are no situations, besides those that can be reached through executing actions. This language is specified by the concepts: categories, predicates and functions and the key concepts for situation calculus are the objects, object's variables, categories, standard predicates and function, as well as micro situations, states, actions, measures, units and time. Using these concepts, it is feasible to model situations, systematize and classify objects and their features, perform analysis of situations and dynamics of their changes and formulate inference rules for the choice of management decisions.

One of the authors' proposal is to consider semantic modeling as one of the directions of semiotic modeling, in which the graphic representation of the developed models predominates, with elements of cognitive graphics. Similarity of basic concepts of situation management and situation calculus makes it possible to integrate these approaches.

The ontology of situation calculus, depicted in Fig. 3, was built with principal concepts of situation calculus and those of situation management, which were described above.

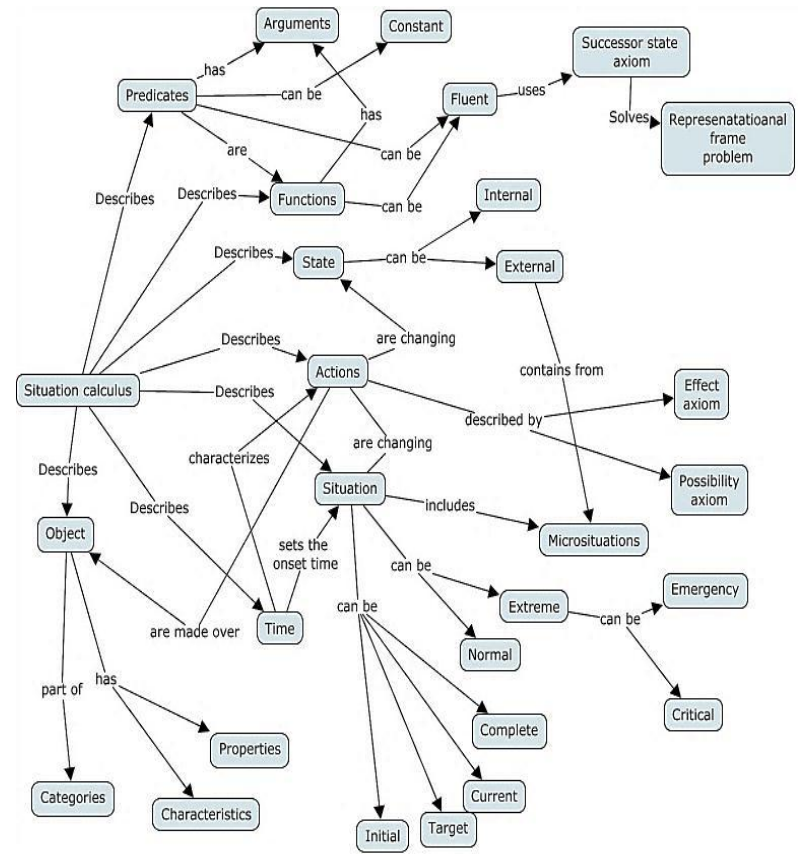

Fig. 3. Ontology of situation calculus

Intersection with concepts of situation management is reflected in situation classification that was included into ontology, as well as in the concepts "object", "action and "state".

Consider some of the language relationships [22], displayed in Fig. 2. They are referred to the component of knowledge description and compared with the concepts of situation calculus language, to be represented through: 


\author{
Relations of naming: \\ $\langle$ object> <has > <name> \\ $\langle$ object $\rangle:=\langle$ physical object $\rangle \mid\langle$ software \\ component $>\mid$ informational component > \\ For this type of relations it's possible to use object \\ characteristics, introduced in situation calculus. \\ Relations of classification: \\ $<$ situation > <has type > <initial $\mid$ transitional $\mid$ target $>$ \\ $<$ control action | measure > <has type > <preventive | \\ operative | liquidation>. \\ Situation calculus aims to classify situations and actions. \\ To classify threats it is possible to use dictionary of \\ names and terms.
}

\section{Spatial relations:}

<situation> <happens at> <location>

To describe this relations it's proposed to use dictionary of names and terms.

\section{Time relations:}

<situation> <happens at> <time>

When describing these relations one can use the concept of time, because in situation calculus it sets the onset time of specific situation.

Tentative analysis shows that in such a way it is possible to consider all proposed relationships and to compare them with the basic concepts of situation calculus language.

\section{Software Complex Semp-TAO}

This complex is based on the knowledge representation model, which combines both classical and modern tools of representation and processing of knowledge: frames, semantic networks, production systems [23]. The main tool of representing declarative knowledge in the environment is the semantic network. The processes of output and processing of information are specified in the form of production systems which work on a semantic network.

Based on this model, the language of representation and processing of knowledge was developed, which is intended for specification of applications in various subject areas and has a rich set of tools [24]. It allows to represent domain concepts in the form of object classes and relationships, enter new data types and specify the required output and processing processes in applications, and includes the tools necessary for creation of user interfaces and work with text files.

Now, we'll take into consideration how with the help of Semp-TAO language it's possible to describe structure of some objects, which are used in decisionmaking support system.
First, create the following base classes:

- commonObject - describes common object in the system. It has following fields: "name" (object's name), "global_id" (object identifier in database), "owner_id" (identifier of the model to which the object belongs) and "inner_id" (object identifier inside of the model).

- commonRelationStruct - describes common relation between objects. It has following fields: "global_incoming_id" (global identifier of the object, where relation starts), "inner_incoming_id" (inner identifier of the object, where relation starts), "global_outer_id" (global identifier of the object, where relation ends), "inner_outer_id" (inner identifier of the object, where relation ends) and "value" (some relation value).

- commonMap - describes common object of the model. It has following fields: "global_id" (model identifier in database), "name" (model name) and "description" (model description).

Now, on the example of ontology, it'll be shown how one can use classes, described above to describe ontology. Preliminarily, we'll create class, which describes ontology concept with following fields: "incoming_relations" (set of object incoming relations) and "outgoing_relations" (set of outgoing relations). This class is inherited from commonObject class. Then, ontology class is described, it consists from single field "objects", which is a set of ontology concepts. Also, this class extends commonMap class.

Thus, one can see that it is possible to describe the concepts of the contingency management language using the Semp-TAO language.

\section{Conclusion}

In this article, main concepts of situation management, semiotic approach and situation calculus were considered. Structure of "Situational polygon" intelligent decision-making support system in energy sector was shown and one of its basic components Contingency Management Language (CML) was briefly considered. Also, two approaches to implementation of CML were presented.

\section{Acknowledgements}

The results provided in the article have been acquired under the partial financial support of RFBR grants № 16-07-00474, № 17-07-01341, № 18-57-81001, № 1807-00714 


\section{References}

1. Momoh J., Smart Grid: Fundamentals of design and analysis (New York, John Wiley and Sons, 2012)

2. Buran A., Nizkodubov G., Pryahin I., Smart Grid Technologies: Trends and Perspectives, MATEC Web Conf. 91010542017

3. Ryan F. Kelly, Adrian R. Pearce, Asynchronous knowledge with hidden actions in the situation calculus, Artificial Intelligence, vol. 221 (2015) 1-35.

4. Giuseppe De Giacomo, Yves Lespérance, Fabio Patrizi, Stavros Vassos, Progression and Verification of Situation Calculus Agents with Bounded Beliefs, Stud Logica, no. 104(4) (2016) 705-739.

5. Lee Joohyung, Palla Ravi, Reformulating the situation calculus and the event calculus in the general theory of stable models and in answer set programming, Journal of Artificial Intelligence Research Vol. 43. (2012) 571-620

6. Ferrein A., Maier C., Mühlbacher C., Niemueller T., Steinbauer G., Vassos S., Controlling Logistics Robots with the Action-Based Language YAGI, in: Kubota N., Kiguchi K., Liu H., Obo T. (eds) Intelligent Robotics and Applications. ICIRA 2016. Lecture Notes in Computer Science, vol 9834. (Springer, Cham, 2016).

7. Vincenzo Corrado, Ilaria Ballarini, Leandro Madrazo, German Nemirovskij, Data structuring for the ontological modelling of urban energy systems: The experience of the SEMANCO project, Sustainable Cities and Society, Volume 14 ( 2015) 223-235.

8. Sabou M., Kovalenko O., Novák P., Semantic Modelling and Acquisition of Engineering Knowledge, in Biffl S., Sabou M. (eds) Semantic Web Technologies for Intelligent Engineering Applications (Springer, Cham, 2016).

9. Joanna Alvarado-Uribe, Arianna Becerril García, Miguel Gonzalez-Mendoza, Rafael Lozano Espinosa, José Martín Molina Espinosa, Semantic Approach for Discovery and Visualization of Academic Information Structured with OAI-PMH, Acta Polytechnica Hungarica 14(3), (2017) 129-148.

10. Liudmila V. Massel, Creation problems of intelligent systems of semiotic type for strategic contingency management in critical infrastructures, Information and mathematical technologies in science and management. №1.(2016) 7-27. (in Russian).

11. 12th World Congress of Semiotics "New Semiotics: Between Tradition and Innovation": abstracts. (Bulgaria. Sofia. New Bulgarian University/Southeast European Center For Semiotic Studies. 2014).

12. Panov A. I., Yakovlev K., Behavior and Path Planning for the Coalition of Cognitive Robots in Smart Relocation Tasks, in Kim J.H., Karray F., Jo J., Sincak P., Myung H. (eds) Robot Intelligence Technology and Applications 4. Advances in Intelligent Systems and Computing, vol 447.(Springer, Cham, 2017).

13. Sarbo J.J., Yang J.H., A Semiotic Approach to Critical Reasoning, in Liu K., Nakata K., Li W., Galarreta D. (eds) Information and Knowledge Management in Complex Systems. ICISO 2015. IFIP Advances in Information and Communication Technology, vol 449. (Springer, Cham. 2015).
14. Pospelov D. A., Situation management principles, Proceedings of the Russian Academy of Sciences Techincal cybernetics, №2, (1971) 10-17. (in Russian).

15. Massel L. V., Massel A. G., Arshinsky V. L., Intelligent IT-enviroment for decision support in research and energy security guaranty of Russia and its regions, Proceedings 13th Workshop on Computer Science and Information Technologies. Vol. 1. (Germany. GarmishPartenkirchen. KIT, 2011) 7-13.

16. Osipov G. S., From situation management to applied semiotics, News of artificial intelligence 6(54) (2002) 2-12.

17. John McCarthy., Situations, actions and causal laws. Technical Report Memo 2 (Stanford University Artificial Intelligence Laboratory, Stanford, CA, 1963).

18. Stuart J. Russell, Peter Norvig, Artificial Intelligence A Modern Approach, 3nd edn. (Prentice Hall, Englewood Cliffs, New Jersey, 2010).

19. Devyatkov V. V., Artificial intelligence systems: Textbook for high schools (M.:Bauman Moscow State Technical University publishing, 2001). (in Russian).

20. Massel A. G., Massel L. V., Situation polygon as intelligent system of semiotic type, in Proceedings of XLIII International conference "Informational technologies in science, education and management" (Irkutsk, MESI SB RAS, 2015) pp. 246-255. (in Russian).

21. Massel L. V., Massel A. G., Language of description and management of knowledge in intelligent system of semiotic type, in Proceedings XX Baikal Allrussian conference "Informational and mathematical technologies in science and management" Vol. 3. (Irkutsk, MESI SB RAS, 2015) pp. 112-124. (in Russian).

22. Massel A. G., Massel L. V., Development of the language of situation management in an intelligent system of semiotic type based on ontological engineering, in Proceedings VI International Scientific Conference OSTIS-2016 (Minsk, Belarus State University of Informatics and Radiotechnics, 2013) pp. 65-70. (in Russian).

23. Zagorulko Yu. A., Popov I. G., A Software Environment based on an Integrated Knowledge Representation Model, in Proc. of the Andrei Ershov Second Internat. Conf. PSI'96 Perspectives of System Informatics (Novosibirsk, Russia, June 25-28, 1996) pp. 300-304.

24. Zagorulko Yu. A., Popov I. G., Knowledge representation language based on the integration of production rules, frames and a subdefinite model, Joint Bull. of the NCC \& IIS. Ser.: Comput. Sci. Iss. 8. (1998) 81-100. 